\title{
Isolation and Identification of Probiotic to Improve Nutritional Value of Diet Supplements
}

\section{Urva Akmal ${ }^{1}$, Muhammad Muneeb Subhani ${ }^{4}$, Shahid Usman ${ }^{2}$, Salman Shabbir Abbasi ${ }^{3}$, Sonyia ${ }^{5}$, Ifra Ghori ${ }^{6}$, Shakira Ghazanfar ${ }^{7 *}$ and Irum Arif ${ }^{7}$}

${ }^{1}$ Department of Environmental Sciences, Fatima Jinnah Women University Rawalpindi, Pakistan

${ }^{2}$ Department of Microbiology, Hazara University Mansehra, Pakistan

${ }^{3}$ Department of Biological Sciences, International Islamic University, Islamabad, Pakistan

${ }^{4}$ Center of Agri Biochemistry and Biotechnology (CABB), University of Agriculture Faisalabad, Pakistan

${ }^{5}$ Department of Biotechnology, Fatima Jinnah women University Rawalpindi, Pakistan

${ }^{6}$ Department of Microbiology, Govt College University Faisalabad, Pakistan

${ }^{7}$ National Institute of Genomics and Agriculture Biotechnology (NIGAB), National Agriculture Research Centre, Islamabad, Pakistan

*Corresponding Author: Shakira Ghazanfar, National Institute of Genomics and Agriculture Biotechnology (NIGAB), National Agricultural Research Centre, Islamabad, Pakistan.

Received: June 26, 2019; Published: July 25, 2019

DOI: $10.31080 /$ ASNH.2019.03.0381

\begin{abstract}
Probiotics are the live microbial feed supplement when administered in adequate amounts confers health benefits in the host. The objective of present research was to isolate the Lactic acid bacteria as potential probiotic. 10 bacterial strains were isolated from pickles and identified based on their morphological and biochemical characteristics. The non-sporulating, non-motile, gram positive, catalase and oxidase negative bacterial strains were selected for further probiotic characterization. All starins showed good probiotic potential. The Pediococcus acidilactici strain showed the best probiotic potential from all other strains. Our results demonstrated that the Pediococcus acidilactici is a unique bacteria for diet supplements. But, future research is required to produce nutritional diet supplements after assessing their advanced probiotic characteristics.
\end{abstract}

Keywords: Probiotics; Pediococcus acidilactici

\section{Introduction}

Assessment of current scenario related to food quality and safety determine that pathogenic microflora have gained resistance towards microbial agents through DNA vertical and horizontal transmission as a result of continual utilization of beneficial microflora [1]. As a result, these pathogenic microflora causes spoilage of food or make them unable for consumption. To resolve this reckless issue, scientists from all over the world are engaged to discover microorganisms (probiotics) proved to be beneficial to feed living organisms.

Probiotics are the live microbial feed additives, which improve the host health by improving gut microbiota. The immunomodu- latory properties of probiotics, however, are not displayed consistently and depend on the strain used, age of animals, and a number of other factors (Ohashi and Ushida 2009) It is advocated, therefore, to search for high-potential health beneficial bacteria from the human or animal microbiota that can be applied in a probiotic-like manner for this purpose. (van Baarlen, Wells., et al. 2013) As mentioned above, similarly to the mechanisms of action of probiotics in humans, the production of large amounts of lactic acid is thought to create an unfavorable environment for certain bacteria, including pathogenic strains of E. coli, Salmonella, or Shigella. Since the adoption of the term DFM (direct-fed microbial; a formulation of viable naturally occurring bacteria, fungi, or yeast) by FDA about two decades ago, there have been gigantic advances and progress 
that have established the beneficial outcomes of exploiting microorganisms and other feed additives in animal diets for improving animal's healthiness and productivity. The use of beneficial microbes in animal diets is appearing to be a promising mean for improved nutrient utilization and increased overall productivity of ruminants. A wide array of bacteria and yeasts have been screened out from the pools of naturally occurring microbes which are capable of positively manipulating and improving the production enhancements in ruminants such as nutrient digestion, nutrition, overall health, and productivity. Numerous DFMs are accessible today that have been rigorously documented for their positive effects on animal's weight gain, rumen development and digestion, restoration of intestinal microflora, producing bacteriocins, competitively reducing opportunistic pathogens, etc. In addition, several DFMs could also produce nutrients and stimulatory growth factors which in turn promote other beneficial microorganisms to flourish within the animal's digestive tract and boost the release of digestive enzymes thereby aiding in the feed digestion by the animal. Moreover, some selected DFM formulations have been proposed to perk up the immune responses as well as metabolism of the animals. Majority of such DFM mainly include lactic acid bacteria, probiotics, and yeasts, since these microbes confer several benefits when added to the animal's diet. These benefits may include stimulation of beneficial microbial growth in the rumen, decrease in ruminal acidosis, stabilization of the rumen $\mathrm{pH}$, positive alteration of ruminal fermentation and end-product production, increase in ruminal propionate concentrations, increased plasma hormones, increase in nutrient flow, efficient nutrient digestibility, enhanced immune responses, and increased milk production Generally, the population densities of rumen bacteria are dominating with the magnitude of $10^{\prime} 10-10^{\prime} 11$, with rumen protozoa prevailing in the range of 10' $5-10^{\prime} 7$ and anaerobic fungi and some facultative anaerobic bacteria falling in the range of 10'4 (McAllister and Cheng 1996) However, these proportions of microbial population may vary significantly or slightly in response to animal diet types such as hay to concentrate rations, concentrate diets, complexity of fibers, pH fluctuations, etc. (Mackie, Gilchrist., et al. 1984)

Therefore, the present study was designed to determine whether probiotics (Lactobacillus) have probiotic potential or not. In this experimental based research, probiotics were isolated from traditional pickles, collected from the Rawalpindi region of Pakistan.
The pickle samples were of carrots (Daucus carota subsp. Sativus), radish (Raphanus raphanistrum subsp. Sativus), garlic (Allium sativumi) and green chilli (Capsicum annuum).

\section{Material and Methods}

Isolation and biochemical characterization of bacterial strains

Isolation of probiotics was done by the procedure given by [2]. Serial dilution was done by transferring $1 \mathrm{ml}$ of brine from sample into $9 \mathrm{ml}$ of autoclaved saline. After that, serial dilution was further done by using this diluent. $0.02 \mathrm{ml}$ from each diluent was spread on MRS media plates and left for 24 hours at 37oC (Table 1). 10 Lactic Acid bacterial strains (PCR99, PCR100, PCR118, PCR119, PCR121, PCR125, PCR130, PCR137, PCR140 AND PCR141) were identified based on their morphological and biochemical characteristics by using the procedure given by [2-4]. Therefore, non-sporulating, non-motile, gram positive, catalase and oxidase negative LAB strains were selected for further probiotic characterization.

\begin{tabular}{|l|c|}
\hline \multicolumn{2}{|c|}{ MRS Agar recipe X amount } \\
\hline Ingredients & Amount (\%) \\
\hline Peptone & 1.0 \\
\hline Beef extract & 1.0 \\
\hline Yeast extract & 0.4 \\
\hline Glucose & 2.0 \\
\hline Sodium acetate & 0.5 \\
\hline Polysorbate 80 & 0.1 \\
\hline Dipotassium hydrogen phosphate & 0.2 \\
\hline Tri ammonium citrate & 0.2 \\
\hline Magnesium sulfate & 0.02 \\
\hline Manganese sulfate & 0.005 \\
\hline Agar & 1.0 \\
\hline
\end{tabular}

Table 1: MRS Agar recipe X amount.

Identification of bacterial strains by 16S rRNA gene sequence

The bacterial starins were identified through 16S rRNA gene sequence by using the commercial service Macrogen Korea. The sequence was identified through EXBio Cloud service. The identified sequence was submitted to NCBI for accession numbers. The phylogenetic tree of the isolated strain was made through MEGA 7. 
Probiotic chaterization of the selective bacterial strains

\section{Antibiotic resistance}

For the determination of the media was prepared for the preparation of Mueller-Hinton plate, we used the standard method. The inoculum was prepared and the MH plates was inoculated in that plates. The antibiotic disc placement was done on these plates and then set them for overnight incubation. The zone of the size was measured through the standard CLSI guidelines.

\section{Results}

These 10 strains were molecularly identified as Lactobacillus plantarum (PCR99, PCR100, PCR125), Lactobacillus. paracasei (PCR118, PCR119, PCR121, PCR140, PCR141), L. brevis (PCR137) and P. acidilactici (PCR130). To utilize these isolated Lactobacillus and Pediococcus strains as probiotics, tolerance to low acidic and salt conditions is crucial. This tolerance makes them able to tolerate gastrointestinal tract severe conditions and function in digestive system. Results of present study shows that Lactobacillus plantarum (PCR100), Lactobacillus paracasei (PCR118) and Pediococcus acidilactici (PCR130) shows best response towards low acidic ( $\mathrm{pH} 3$ and $\mathrm{pH} 4)$ and bile salt conditions (0.3\% and $0.5 \%)$ and show highest cell surface hydrophobicity values $84.62 \%, 82.39$ and $86.12 \%$. Pediococcus acidilactici showed the best results from all strains. All the strains show different enzymatic potential responses indicating that this property is strain-dependent. Furthermore, study relating to their antibiotic susceptibility done by using the procedure given by [5] resulted that Lactobacillus plantarum (PCR100) is resistant to Ampicillin, tetracycline, Kanamycin, polymyxin B, Metronidazole, Amoxycillin, Nalidixic Acid, Cefuroxime, Vancomycin. While, Lactobacillus paracasei (PCR118) showed resistance towards Cefuroxime, Amoxycillin, Metronidazole, Penicillin G, Ampicillin, Polymyxin B, Bacitracin. Besides, Pediococcus acidilactici (PCR130) showed resistance towards Metronidazole, Amoxycillin and Penicillin G. Antibiotic resistance among probiotic bacterial strains is desirable or not is a debated since some scientists claimed that they can pass their resistance to pathogenic microflora and make them more detrimental for health. On contrary to this, other scientists have different point of view that probiotics are healthy as they feed beneficial digestive microflora which are killed and destroyed by using antibiotics.
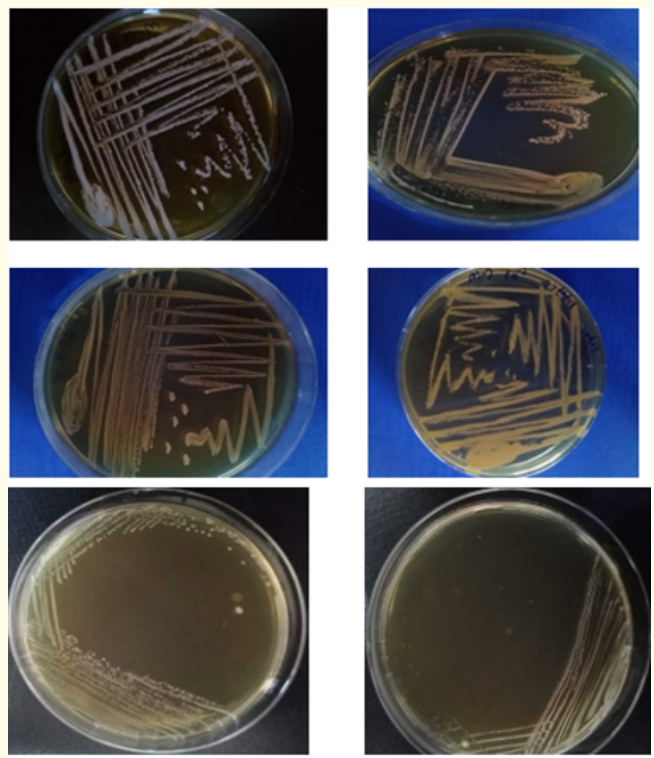

Figure 1: Colony morphologies of bacterial isolates on MRS agar media.
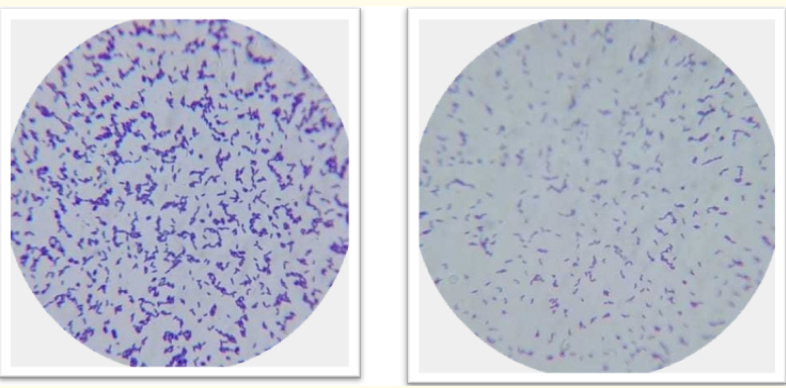

Figure 2: Microscopic observation of gram staining isolated bacterial species.
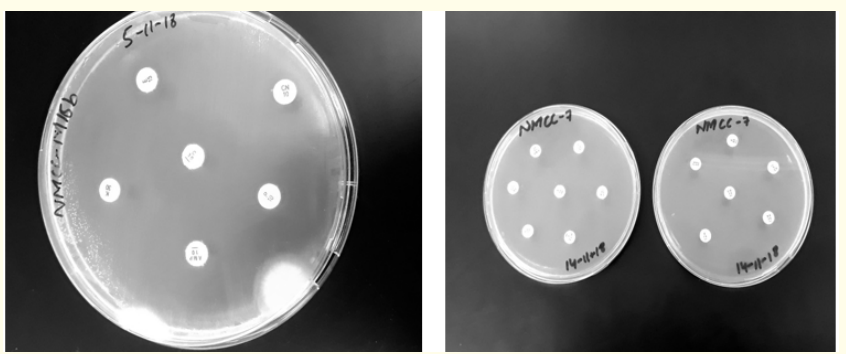

Figure 3: Antibiotic susceptibility of the Pediococcus acidilactici. 


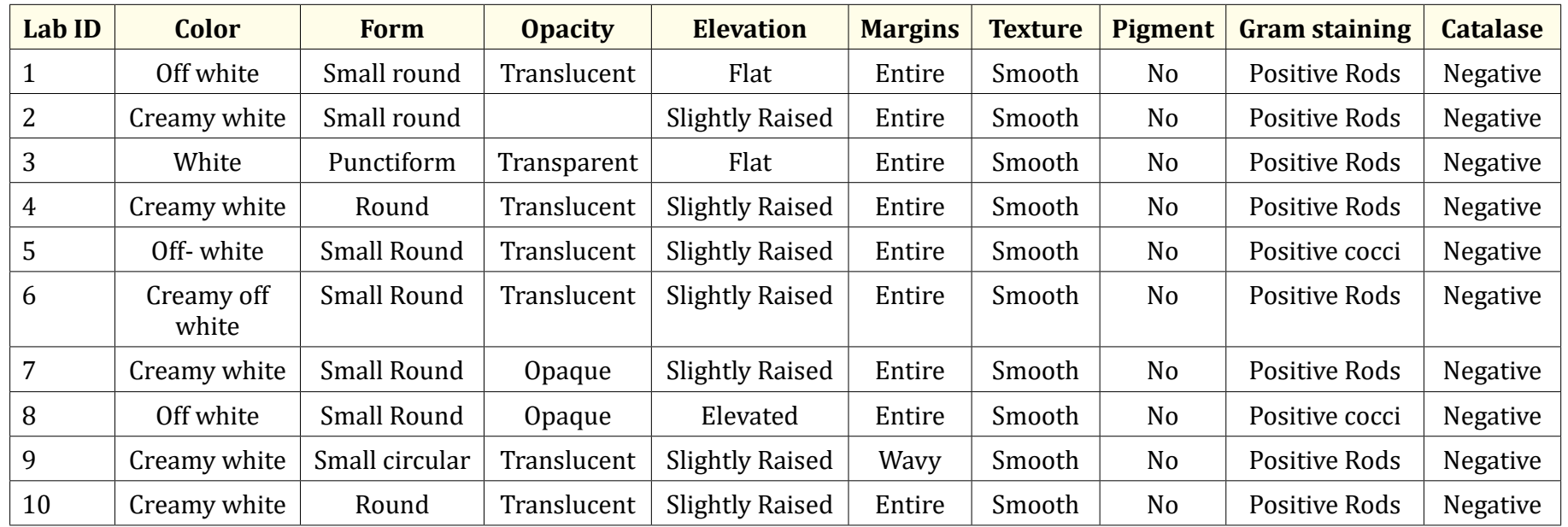

Table 2: Morphological Characterization Samples.

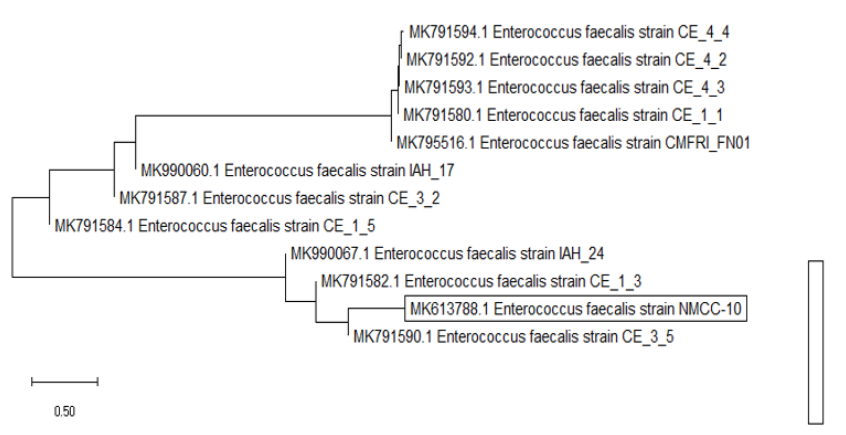

Figure 4: Phylogenetic tree of NMCC-10 bacterial strain.

\section{Conclusion}

Results of the present study concluded that Lactobacillus Paracasei (PCR118), Lactobacillus plantarum (PCR100) and Pediococcus acidilactici (PCR130) are best for their utilization to produce nutritional supplements for human beings and animal as they showed beneficial and desirable responses to low acidic and bile salt conditions, possess highest hydrophobicity values and displayed sensitivity towards antibiotics. Therefore, future research is required to produce nutritional diet supplements after assessing their advanced probiotic characteristics.

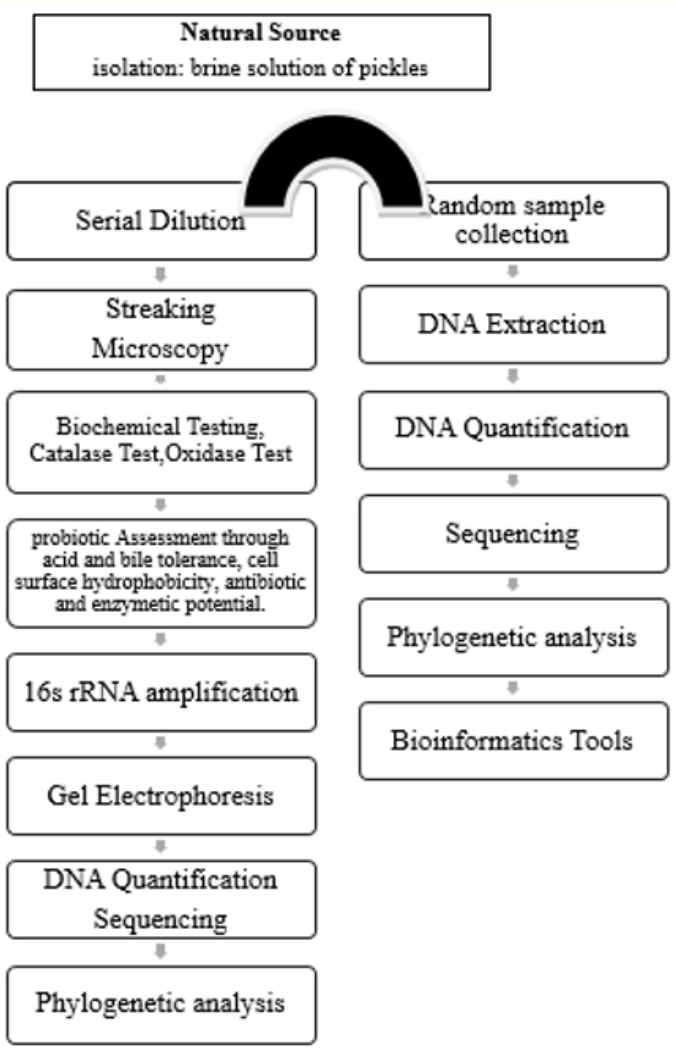

Figure 5: Isolation and Characterization of Probiotic Lactic Acid Bacterial Strains From Traditionally Fermented Pickles. 


\section{Bibliography}

1. Founou., et al. "Antibiotic Resistance in the Food Chain: A Developing Country-Perspective". Frontiers in Microbiology 7 (2016).

2. Monika Savitri., et al. "Isolation and Characterization of Lactic Acid Bacteria from Traditional Pickles of Himachal Pradesh, India". Journal of Food Science and Technology 54 (2017a): 1945-1952.

3. "Bergey's manual of determinative bacteriology (7th Ed.)". American Journal of Public Health and the Nations Health 54 (1964): 544.

4. Ghazanfar S., et al. "Effect of indigenously isolated saccharomyces cerevisiae probiotics on milk production, nutrient digestibility, blood chemistry and fecal microbiota in lactating dairy cows". Journal of Animal and Plant Sciences (2018).

5. "Isolation and Characterization of Lactic Acid Bacteria from Traditional Pickles of Himachal Pradesh, India." Journal of Food Science and Technology 54 (2017b): 1945-1952.

Volume 3 Issue 8 August 2019

(C) All rights are reserved by Shakira Ghazanfar., et al. 